www.jmscr.igmpublication.org

Index Copernicus Value: 79.54

ISSN (e)-2347-176x ISSN (p) 2455-0450

crossref DOI: https://dx.doi.org/10.18535/jmscr/v7i5.130

Journal Of Medical Science And Clinical Research

IGM Publication

An official Publication of IGM Publication

\title{
Palliative Emergency mediastinal tracheostomy in carcinoma thyroid
}

Authors

\section{Dr Amit Kumar Agarwal, Mch CTVS ${ }^{1}$, Dr Sunil Dhar, Mch CTVS ${ }^{2}$,} Dr Anubhav Gupta ${ }^{3}$, Dr Ajit Kumar Padhy ${ }^{* *}$

${ }^{1,2}$ Dept of Cardiothoracic Surgery, VMMC and Safdarjung Hospital, New Delhi.110049

${ }^{3}$ Professor and H.O.D, Dept of Cardiothoracic Surgery, VMMC and Safdarjung Hospital, New Delhi.110049

${ }^{4}$ Asst. Prof., CTVS, Dept of Cardiothoracic Surgery, VMMC, and Safderjung Hospital New Delhi 110049

*Corresponding Author

Dr Ajit Kumar Padhy

Asst. Prof., CTVS, Dept of Cardiothoracic Surgery, VMMC, and Safderjung Hospital New Delhi 110049

\begin{abstract}
Emergency mediastinal tracheostomy is life saving in patients with unresectable cervical tumors, in whom the site of the tracheostomy lies in the upper mediastinum in the tumor free area. Radiotherapy increases the risk due to substernal adhesion and presence of vascular structure make the procedure complex in this area. Careful nibbling of manubrium and left sternoclavicular joint with lateral traction of proximal 1-2cm of innominate artery after their origin from aorta gives a good exposure of substernal segment of trachea.
\end{abstract}

\section{Introduction}

Medullary carcinoma of thyroid involving tracheal lumen can cause life threatening respiratory distress. Establishing a patent airway by standard tracheostomy is not appropriate as the site is obscured by tumour tissue .To establish an airway in the mediastinal segment of trachea by Partial splitting sternotomy under local anaesthesia pose a significant challenge in a post radiotherapy patient due to extensive substernal inflammation and adhesion. In such case, nibbling of manubrium sterni and left sternoclavicular joint with lateral traction of the proximal innominate artery gives a good exposure of the substernal segment of trachea.

\section{Case Report}

A 35 year old male, a known case of medullary carcinoma of thyroid with tracheal infiltration underwent multiple cycles of radiotherapy, debulking of tumor and bilateral modified radical neck dissection presented to emergency room with stridor and tachypnea (respiratory rate-45/min). His room air oxygen saturation was $89 \%$ and with high flow oxygen $(8 \mathrm{Ltr} / \mathrm{min})$ the oxygen saturation increased to $98 \%$.A palliative emergency tracheostomy planned under local anaesthesia.

Initially, the incision was given in the midline at the level of upper tracheal rings, but due to adhesions and inflammatory tissue at the site the standard tracheostomy was not possible to carry out. Therefore, the incision was further extended 
over the manubrium in the mid line up to sternal angle. The retrosternal space was difficult to reach due to dense adhesion. Hence instead of partial upper median sternotomy we preferred to resect a part of manubrium and left sternoclavicular joint lying over the trachea with the help of a bone nibbler. Trachea could be reached with blunt finger dissection after retracting the Innominate artery to the right. (Fig-1).A $6 \mathrm{~mm}$ Size cuffed tracheostomy tube passed through the anterior tracheal stoma and the skin layer is closed with interrupted nylon suture. Patient was relieved of stridor and room air oxygen saturation improved to $98 \%$ with no tachypnea.

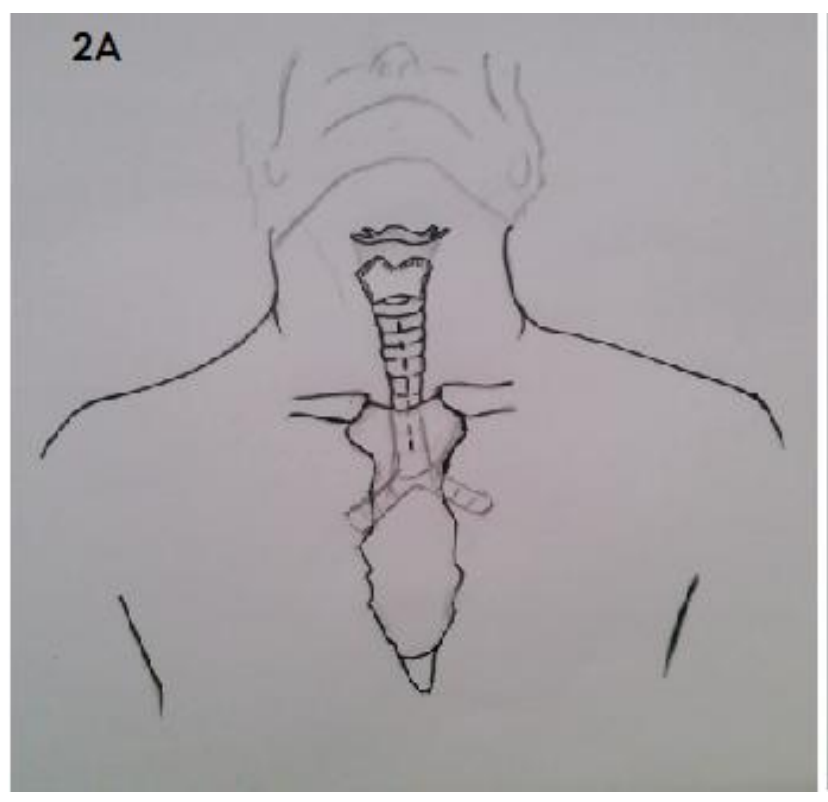

Figure-2A: schematic illustration of cervical incision in the mid line for standard tracheostomy extended to chest over the manubrium for mediastinal tracheostomy. Figure -2B: Schematic illustration of mediastinal tracheostomy showing the mediastinal segment of trachea and its relation with great vessels after resection of manubrium sterni and left sternoclavicular joint. Dotted circle over the anterior tracheal wall is the site of tracheostomy.

\section{Discussion}

Cervical tumors with local advancement up to the thoracic inlet are known to cause mechanical airway obstruction. Historically, curative mediastinal terminal tracheostomy involving cervical tracheal excision has been associated with high operative mortality, generally secondary to mediastinal infection and rupture of the innominate vessels. Simple palliative mediastinal
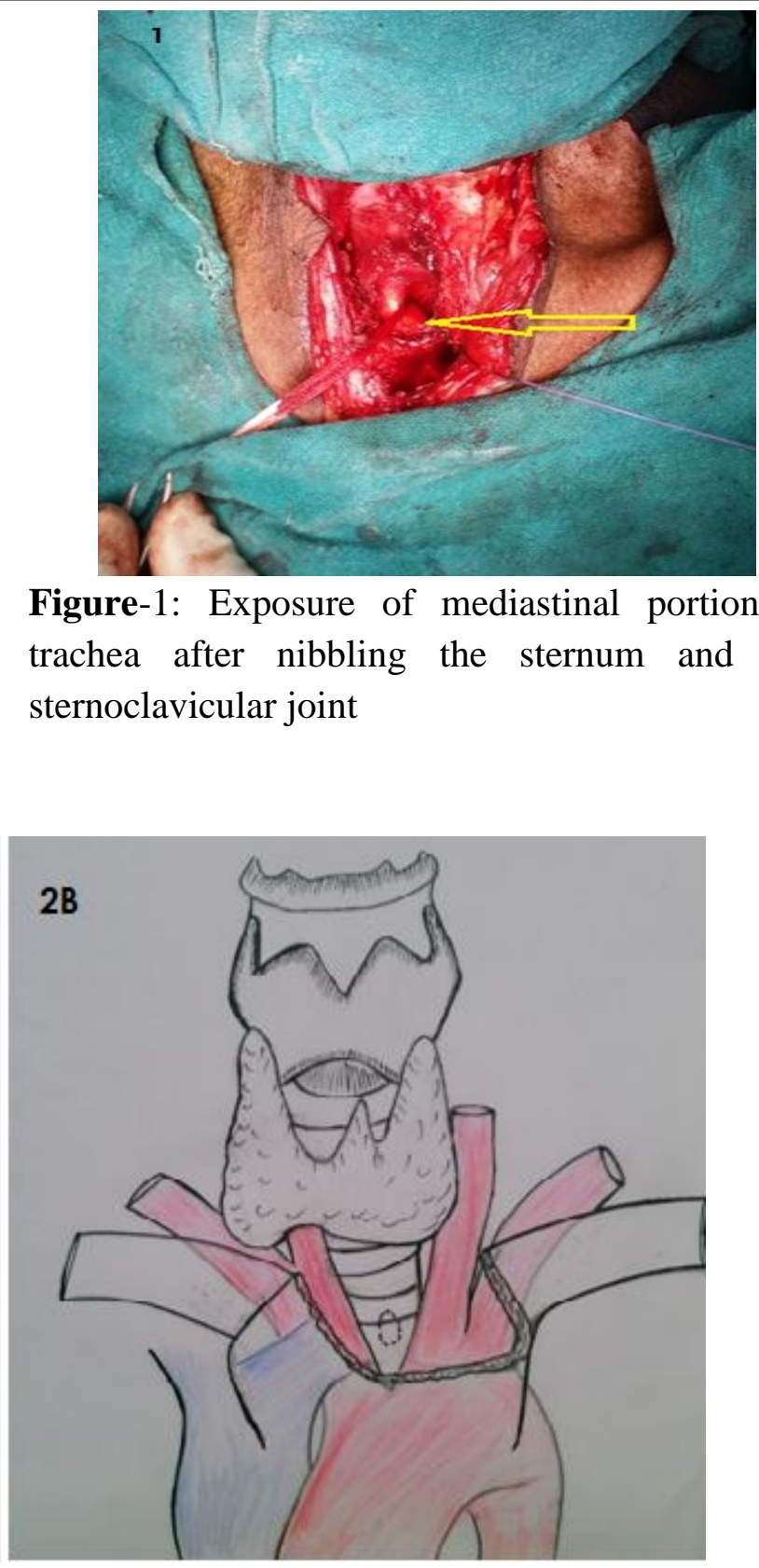

Figure-1: Exposure of mediastinal portion of trachea after nibbling the sternum and left sternoclavicular joint 
permits a safe access to the superior mediastinum in emergency.

\section{Conclusion}

Emergency mediastinal tracheostomy improves the respiratory symptoms in patients with cervical malignancy involving the trachea where standard tracheostomy is not possible due to malignant tissue. It is prudent to nibble the bone where partial splitting sternotomy pose a surgical challenge due to prior neck irradiation

\section{Compliance with Ethical Standards}

This study was not funded

This article does not contain any studies with animals performed by any of the authors

Informed consent was obtained from the individual participant included in the study

\section{References}

1. Orringer MB. Anterior mediastinal tracheostomy with and without cervical exenteration. Ann Thorac Surg 1992;54:628-37.

2. Conti M, Benhamed L, Mortuaire G, et al. Indications and results of anterior mediastinal tracheostomy for malignancies. Ann Thorac Surg 2010;89: $1588-96$.

3. Gomes MN, Kroll S, Spear SL. Mediastinal tracheostomy. Ann Thorac Surg 1987;43:539-43.

4. Yasumichi Yamamoto, Toshiya Toyazaki, Shinji Kosaka Simple Palliative Mediastinal Tracheostomy (Ann Thorac Surg 2013;95:2185-7). 